\title{
A note on the speed of hereditary graph properties
}

\author{
Vadim V. Lozin* \\ DIMAP and Mathematics Institute \\ University of Warwick, Coventry CV4 7AL, UK \\ V.Lozin@warwick.ac.uk \\ Colin Mayhill \\ Victor Zamaraev ${ }^{\dagger}$ \\ Mathematics Institute \\ University of Warwick, Coventry CV4 7AL, UK \\ C.R.Mayhill@warwick.ac.uk \\ University of Nizhny Novgorod, Russia \\ Viktor.Zamaraev@gmail.com \\ Submitted: Jan 27, 2011; Accepted: Jul 27, 2011; Published: Aug 5, 2011 \\ Mathematics Subject Classification: 05C30
}

\begin{abstract}
For a graph property $X$, let $X_{n}$ be the number of graphs with vertex set $\{1, \ldots, n\}$ having property $X$, also known as the speed of $X$. A property $X$ is called factorial if $X$ is hereditary (i.e. closed under taking induced subgraphs) and $n^{c_{1} n} \leq X_{n} \leq n^{c_{2} n}$ for some positive constants $c_{1}$ and $c_{2}$. Hereditary properties with the speed slower than factorial are surprisingly well structured. The situation with factorial properties is more complicated and less explored, although this family includes many properties of theoretical or practical importance, such as planar graphs or graphs of bounded vertex degree. To simplify the study of factorial properties, we propose the following conjecture: the speed of a hereditary property $X$ is factorial if and only if the fastest of the following three properties is factorial: bipartite graphs in $X$, co-bipartite graphs in $X$ and split graphs in $X$. In this note, we verify the conjecture for hereditary properties defined by forbidden induced subgraphs with at most 4 vertices.
\end{abstract}

Keywords: Hereditary class of graphs; Speed of hereditary properties; Factorial class

\footnotetext{
${ }^{*}$ Research of this author was supported by the Centre for Discrete Mathematics and Its Applications (DIMAP), University of Warwick.

${ }^{\dagger}$ Research of this author was supported by RFFI, project number 11-01-00107-a and by FAP "Research and educational specialists of innovative Russia", project number 2010-1.3.1-111-017-012
} 


\section{Introduction}

A graph property is an infinite class of graphs closed under isomorphism. A property is hereditary if it is closed under taking induced subgraphs. Given a hereditary property $X$, we write $X_{n}$ for the number of graphs in $X$ with vertex set $\{1,2, \ldots, n\}$. Following [5], we call $X_{n}$ the speed of the property $X$. In [1], it was proved that for any infinite hereditary class $X$ different from the class of all graphs,

$$
\lim _{n \rightarrow \infty} \frac{\log _{2} X_{n}}{\left(\begin{array}{l}
n \\
2
\end{array}\right)}=1-\frac{1}{k(X)},
$$

where $k(X)$ is a natural number called the index of the class $X$. To define this notion, let us denote by $\mathcal{E}_{i, j}$ the class of graphs whose vertices can be partitioned into at most $i$ independent sets and $j$ cliques. In particular,

- $\mathcal{E}_{2,0}$ is the class of bipartite graphs,

- $\mathcal{E}_{1,1}$ is the class of split graphs,

- $\mathcal{E}_{0,2}$ is the class of graphs complement to bipartite.

Then the index $k(X)$ of a class $X$ is the maximum $k$ such that $X$ contains a class $\mathcal{E}_{i, j}$ with $i+j=k$. Now let us extend this definition by assuming that the index of every finite hereditary class is 0 , and the index of the class of all graphs equals infinity. With this extension, the family of all hereditary classes is partitioned into countable number of subsets each of which consists of classes with the same index. Moreover, the classes $\mathcal{E}_{i, j}$ with the same value of $i+j$ are the only minimal classes in the respective subset. In particular, for $k=2$, there are exactly three minimal classes: bipartite, complements of bipartite, and split graphs. Therefore, an infinite hereditary class of graphs has index 1 if and only if it contains none of the three listed classes. The classes of index 1 have been called in [2] unitary.

The family of unitary classes is of special interest, since it contains many classes of theoretical and practical importance, such as interval graphs, permutation graphs, chordal bipartite graphs, line graphs, forests, threshold graphs, all classes of graphs of bounded vertex degree, of bounded clique-width [4], all proper minor-closed graphs classes (including planar graphs) [12], etc. In order to provide a differentiation of the unitary classes in accordance with their size, let us introduce the following definition: two graph classes $X$ and $Y$ will be called isometric if there are positive constants $c_{1}, c_{2}$ and $n_{0}$ such that $Y_{n}^{c_{1}} \leq X_{n} \leq Y_{n}^{c_{2}}$ for any $n>n_{0}$. Clearly the isometricity is an equivalence relation. The equivalence classes of this relation are called layers.

All finite classes of graphs constitute a single layer, and all classes of index greater than 1 also constitute a single layer. Between these two extremes lies the family of unitary classes, and it consists of infinitely many layers. The first four lower layers in this family have been distinguished in [13]:

- constant layer contains classes $X$ with $\log _{2}\left|X_{n}\right|=O(1)$, 
- polynomial layer contains classes $X$ with $\log _{2}\left|X_{n}\right|=\Theta\left(\log _{2} n\right)$,

- exponential layer contains classes $X$ with $\log _{2}\left|X_{n}\right|=\Theta(n)$,

- factorial layer contains classes $X$ with $\log _{2}\left|X_{n}\right|=\Theta\left(n \log _{2} n\right)$.

Independently, the same result was obtained by Alekseev in [2]. Moreover, Alekseev provided the first four layers with the description of all minimal classes and proposed a structural characterization of the classes in the first three layers (some more involved results can be found in $[5,6]$ ). This characterization shows that the classes in the three lower layers have a rather simple structure. In particular, for any exponential class $X$ there is a constant $c$ such that the vertices of any graph in $X$ can be partitioned into at most $c$ subsets so that each of the subsets is either a clique or an independent set, and between any two of them there are either all possible edges or none of them.

The factorial layer is substantially richer. It contains most of the unitary classes mentioned above (the unique exception in the above list is the class of chordal bipartite graphs, which is superfactorial [14]). However, no complete structural characterization is available for this layer. As a step toward this characterization, we propose the following conjecture.

Conjecture $A$ hereditary graph property $X$ is factorial if and only if the fastest of the following three properties is factorial: $X \cap \mathcal{E}_{2,0}, X \cap \mathcal{E}_{1,1}, X \cap \mathcal{E}_{0,2}$.

This conjecture is suggested by the exceptional role of the three minimal non-unitary classes in the study of lower layers of hereditary properties. In particular, all minimal classes in the first four layers are subclasses of bipartite, co-bipartite or split graphs. Therefore, if we replace in the conjecture the factorial layer by any of the first three lower layers, it becomes a valid statement. For the factorial layer, only one part of the conjecture is known to be true (the "only if" part), since all minimal factorial classes are subclasses of bipartite, co-bipartite or split graphs. The three minimal factorial classes of bipartite graphs are [2]:

- $P^{1}=\operatorname{Free}\left(K_{3}, K_{1,2}\right)$ (the notations are given below), the class of graphs of vertex degree at most 1 ,

- $P^{2}$, the class of "bipartite complements" of graphs in $P^{1}$, i.e. the class of bipartite graphs in which every vertex has at most one non-neighbor in the opposite part,

- $P^{3}=\operatorname{Free}\left(C_{3}, C_{5}, 2 K_{2}\right)$, the class of $2 K_{2}$-free bipartite graphs, also known as chain graphs for the property that the neighborhoods of vertices in each part form a chain.

The complements of graphs in $P^{1}, P^{2}, P^{3}$ form the three minimal factorial classes of co-bipartite graphs. The remaining three minimal factorial classes also are closely related to $P^{1}, P^{2}, P^{3}$. To reveal this relationship, let us observe that by creating a clique in one of the parts of a bipartite graph we obtain a split graph. By applying this operation to graphs in $P^{1}, P^{2}, P^{3}$, we transform these classes into subclasses of split graphs, which are precisely the three remaining minimal factorial classes. For instance, the class $P^{3}$ 
transforms in this way into the subclass of split graphs known as threshold graphs, or equivalently, $\left(2 K_{2}, C_{4}, P_{4}\right)$-free graphs.

To verify the "if" part of the conjecture, we initiate a systematic study a factorial properties based on their induced subgraph characterization. It is well-known (and not difficult to see) that a graph property $X$ is hereditary if and only if it can be described by a set of forbidden induced subgraphs. In this paper, we show that the above conjecture is true for all properties defined by forbidden induced subgraphs with at most 4 vertices, which is the main result of the paper. The organization of the paper is as follows. In the rest of this section we introduce basic notations. In Section 2, we verify the conjecture for all factorial classes defined by forbidden induced subgraphs on at most 4 vertices with one exception. The only exception is the class of $\left(K_{1,3}, C_{4}\right)$-free graphs. We study this class in Section 3, where we prove that this class is factorial.

We use the following notations. For a set of graphs $M$ we denote by $\operatorname{Free}(M)$ the class of graphs containing no induced subgraphs isomorphic to graphs in the set $M$ and call the graphs in this class $M$-free.

For a graph $G$, we denote by $V(G)$ and $E(G)$ the vertex set and the edge set of $G$ respectively. The neighborhood $N(v)$ of a vertex $v \in V(G)$ is the set of vertices adjacent to $v$. If $N(v)$ is a clique, then $v$ is a simplicial vertex. The subgraph of $G$ induced by a set $U \subseteq V(G)$ is denoted $G[U]$, and $G \backslash U$ stands for $G[V(G) \backslash U]$. As usual, $C_{n}, P_{n}$ and $K_{n}$ denote the cycle, the path and the complete graph on $n$ vertices respectively. Also, by $K_{n, m}$ we denote a complete bipartite graph with parts of size $n$ and $m$. An $n$-wheel $W_{n}$ is a graph with $n+1$ vertices obtained from a cycle $C_{n}$ by adding a dominating vertex, i.e. a vertex adjacent to every vertex of the cycle. The complements of a graph $G$ is denoted $\bar{G}$ and is called co- $G$.

\section{Classes of graphs defined by 4-vertex forbidden in- duced subgraphs}

To avoid triviality, we do not forbid graphs on two vertices. Graphs with at least three vertices will be called non-trivial.

There are four graphs on three vertices (triangle $K_{3}$, path $P_{3}$ and their complements) and eleven graphs on four vertices (listed in Figure 1).

In our analysis of classes defined by forbidden induced subgraphs we will use the following two results.

Theorem 1. [4] Every class of graphs of bounded clique-width is (at most) factorial.

Theorem 2. (see e.g. [3]) The class of $C_{4}$-free bipartite graphs is superfactorial.

In particular, from Theorem 2 we derive the following necessary condition for a class $\operatorname{Free}(M)$ to be factorial (note that the class $\operatorname{Free}\left(C_{3}, C_{4}\right)$ contains all $C_{4}$-free bipartite graphs). 


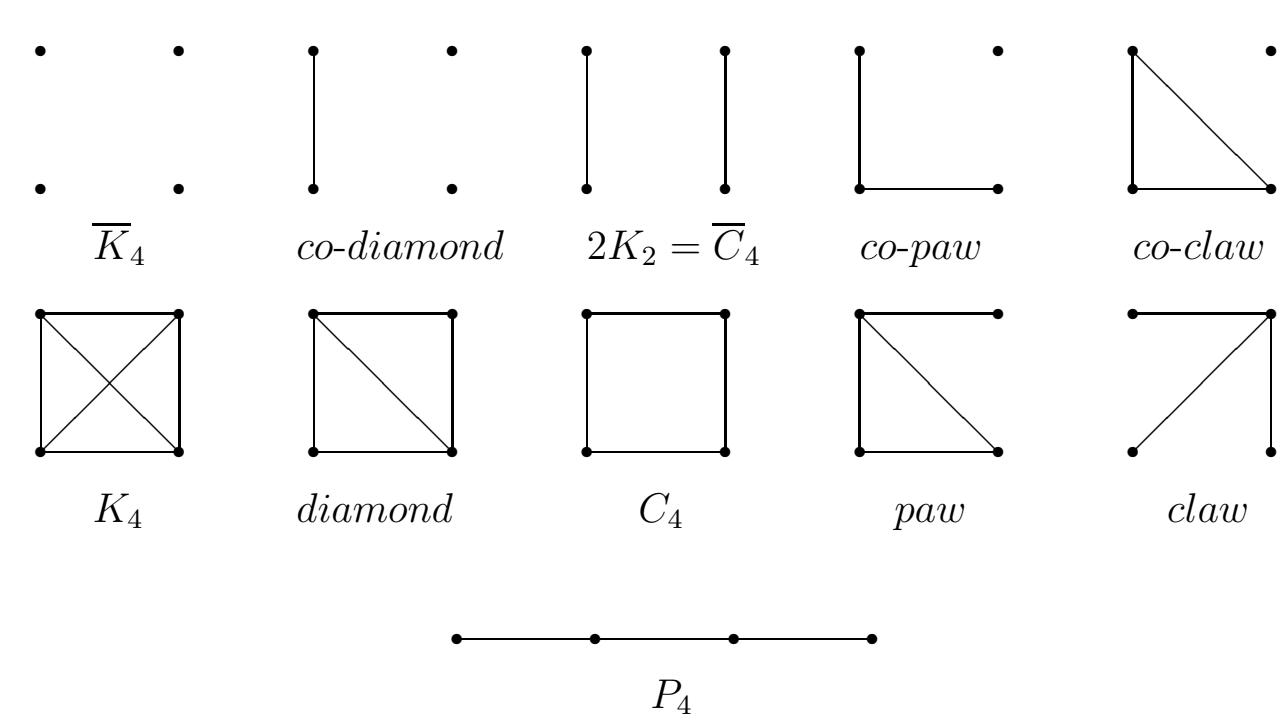

Figure 1: All graphs on four vertices

Theorem 3. Let $M$ be a set of graphs such that

- either $M \cap \mathcal{E}_{0,2}=\emptyset$

- or $M \cap \mathcal{E}_{2,0}=\emptyset$

- or $M \cap \mathcal{E}_{1,1}=\emptyset$

- or $M \cap \operatorname{Free}\left(C_{3}, C_{4}\right)=\emptyset$

- or $M \cap \operatorname{Free}\left(\bar{C}_{3}, \bar{C}_{4}\right)=\emptyset$,

then Free $(M)$ is superfactorial.

Let us call the five classes listed in the theorem critical. In what follows, we show that if $M$ contains a graph in each of the critical classes and each graph in $M$ has at most four vertices, then $\operatorname{Free}(M)$ is (at most) factorial.

There is just one maximal graph contained in all five critical classes, namely a $P_{4}$. It is known (see e.g. [7]) that the clique-width of $P_{4}$-free graphs is at most 2. Together with Theorem 1 this gives a factorial upper bound for the class Free $\left(P_{4}\right)$. The class $\operatorname{Free}\left(P_{3}\right)$ contains $P^{1}$ (one of the minimal factorial classes), which gives a lower bound. Therefore,

Theorem 4. The class Free $(G)$ is factorial if and only if $G$ is a non-trivial induced subgraph of $P_{4}$.

From now on, we assume that none of the forbidden graphs is an induced subgraph of $P_{4}$. This leaves us with 12 non-trivial graphs with at most four vertices none of which is 
an induced subgraph of $P_{4}$. It is not difficult to see that each of them belongs to exactly three of the five critical classes. We divide the set of these 12 graphs into four types according to the critical classes they belong to:

(1) $\bar{K}_{3}, \bar{K}_{4}$, co-diamond, co-paw, claw belong to $\mathcal{E}_{2,0}, \mathcal{E}_{1,1}, \operatorname{Free}\left(C_{3}, C_{4}\right)$.

(2) $K_{3}, K_{4}$, diamond, paw, co-claw belong to $\mathcal{E}_{0,2}, \mathcal{E}_{1,1}$, Free $\left(\bar{C}_{3}, \bar{C}_{4}\right)$.

(3) $2 K_{2}$ belongs to $\mathcal{E}_{2,0}, \mathcal{E}_{0,2}$, Free $\left(C_{3}, C_{4}\right)$.

(4) $C_{4}$ belongs to $\mathcal{E}_{2,0}, \mathcal{E}_{0,2}$, Free $\left(\bar{C}_{3}, \bar{C}_{4}\right)$.

Thus, a necessary condition for the class $\operatorname{Free}(M)$ to be (at most) factorial is that the set $M$ contains at least two graphs: a graph of type (1) and a graph of type (2) or (4) (or their complements). In what follows we show that this condition is also sufficient. Up to symmetry and complementary arguments, we have 20 classes to analyze. Three of them can be easily ruled out by the following observation:

- for any $m$ and $n$, the class $F r e e\left(K_{m}, \bar{K}_{n}\right)$ contains finitely many graphs due to Ramsey Theorem.

It is not difficult to verify that the remaining 17 classes are at least factorial, since each of them contains one of the minimal factorial classes. Now we turn to upper bounds.

First, we refer to some known results. In particular, in [7] it was proved that the following graph classes and their complements have bounded clique-width and hence are at most factorial:

- $\operatorname{Free}\left(K_{4}, c o-p a w\right) \supset \operatorname{Free}\left(K_{3}, c o-p a w\right)$,

- Free $\left(K_{4}\right.$, co-diamond $) \supset \operatorname{Free}\left(K_{3}\right.$, co-diamond $)$,

- Free (diamond, $\left.2 K_{2}\right) \supset \operatorname{Free}\left(K_{3}, 2 K_{2}\right)$,

- $\operatorname{Free}($ paw, claw $) \supset \operatorname{Free}\left(K_{3}\right.$, claw $)$,

- Free(diamond,co-diamond),

- Free(diamond, co-paw),

- Free $\left(\right.$ paw, $\left.2 K_{2}\right)$,

- Free(paw, co-paw),

- Free(co-claw, claw),

This reduces the analysis to the following 4 classes:

- $\operatorname{Free}\left(K_{4}\right.$, claw $)$, 
- $\operatorname{Free}\left(K_{4}, 2 K_{2}\right)$,

- Free(diamond, claw),

- Free (co-claw, $\left.2 K_{2}\right)$.

Theorem 5. The classes Free $\left(K_{4}\right.$, claw), Free $\left(K_{4}, 2 K_{2}\right)$, Free(diamond, claw) are factorial.

Proof. The lower bound follows from the fact that each of these classes contains at least one minimal factorial class. For the upper bound we observe that

- the maximum vertex degree of graphs in Free $\left(K_{4}\right.$, claw $)$ is bounded by 5 , since the neighborhood of each vertex $v$ is $K_{3}$-free (else $v$ belongs to a $K_{4}$ ) and $\bar{K}_{3}$-free (else $v$ is the center of a claw). Therefore, there are at most $n^{5 n}$ graphs with vertex set $\{1, \ldots, n\}$ in the class $\operatorname{Free}\left(K_{4}\right.$, claw).

- the chromatic number of a $2 K_{2}$-free graph $G$ is bounded by $(\underset{2}{\omega(G)+1})$ [15], where $\omega(G)$ is the size of a maximum clique in $G$. Therefore, the vertices of a $\left(K_{4}, 2 K_{2}\right)$ free graph $G$ can be partitioned into at most six independent sets. Each pair of the independent sets induces a $2 K_{2}$-free bipartite graph. Therefore, the edges of $G$ can be partitioned into at most 15 graphs each of which belongs to a factorial class (i.e. $\left.P^{3}\right)$, which gives a factorial upper bound on the number of $n$-vertex labeled graphs in $\operatorname{Free}\left(K_{4}, 2 K_{2}\right)$.

- Free(diamond,claw) is a subclass of the class of line graphs, which is factorial (see e.g. [8]). Independently, a factorial upper bound on the number of graphs in the class Free(diamond, claw) can be obtained by observing that each vertex of a graph in this class belongs to at most two maximal cliques, which shows that the number of $n$ vertex graphs in this class is at most $n^{2 n}$.

The class Free (co-claw, $2 K_{2}$ ) also is factorial, but the proof is more complicated and we postpone it till the next section. Summarizing the above discussion, we obtain the following conclusion.

Theorem 6. Let $M$ be a set of graphs with at most four vertices such that Free $(M)$ is at least factorial (i.e. contains one of the minimal factorial classes). Then Free $(M)$ is factorial if and only if it contains none of the five critical classes $\mathcal{E}_{0,2}, \mathcal{E}_{2,0}, \mathcal{E}_{1,1}, F r e e\left(C_{3}, C_{4}\right)$, $\operatorname{Free}\left(\bar{C}_{3}, \bar{C}_{4}\right)$. 


\section{3 (Claw, Square)-free graphs}

In the previous section we analyzed classes defined by forbidden induced subgraphs with at most four vertices and revealed all factorial classes in this family with one exception, the class Free (co-claw, $2 K_{2}$ ). In this section, we study complements of graphs in Free(coclaw, $2 K_{2}$ ), i.e. graphs which are claw-free and $C_{4}$-free. It is not difficult to see that the intersection of this class with each of the three minimal non-unitary classes is factorial. Therefore, according to the conjecture in the introduction, this class is factorial too. Below we prove this fact. The proof is based on some structural characterizations of claw-free graphs that can be found in the literature. In particular, the following theorem was proved in $[11]$.

Theorem 7. A graph is claw-free and 4-wheel-free if and only if the neighborhood of each vertex is either the complement of a chain graph or a graph obtained from $C_{5}$ or $W_{5}$ by duplication of some of its vertices (i.e. by substituting the vertices with cliques).

Obviously, Free $\left(K_{1,3}, C_{4}\right)$ is a subclass of Free $\left(K_{1,3}, W_{4}\right)$. Therefore, by Theorem 7 , the neighborhood of each vertex of a $\left(K_{1,3}, C_{4}\right)$-free graph induces either the complement of a chain graph or a graph obtained from $C_{5}$ or $W_{5}$ by duplication of some of its vertices. First of all, let us show that without loss of generality we can reduce our analysis to the case when the neighborhood of each vertex of a $\left(K_{1,3}, C_{4}\right)$-free graph induces the complement of a chain graph.

Let $G$ be a $\left(K_{1,3}, C_{4}\right)$-free graph and assume it contains a vertex $x$ whose neighborhood induces a graph obtained from a $C_{5}$ by duplicating some of its vertices. Denote $A=N(x)$ and $B=V(G) \backslash(A \cup\{x\})$. We denote the cliques substituting the vertices of the $C_{5}$ by $A_{i}, i=0, \ldots, 4$ with two cliques being adjacent if and only if their indexes differ by exactly 1 (all additions are taken mod 5). We will show that in order to describe the graph we need to know $G \backslash\{x\}$ and only one neighbor of $x$ in each of the cliques. To this end, let us prove the following lemma.

Lemma 1. Let $a_{i} \in A_{i}$ for $i=0, \ldots, 4$, then $N\left(a_{i-1}\right) \cap N\left(a_{i+1}\right)=A_{i} \cup\{x\}$.

Proof. Without loss of generality, let $i=2$. It is clear that $N\left(a_{1}\right) \cap N\left(a_{3}\right) \cap A=A_{2}$. Now we show that $N\left(a_{1}\right) \cap N\left(a_{3}\right) \cap B=\emptyset$. Suppose not: then $x, a_{1}, a_{3}$ and any vertex from $N\left(a_{1}\right) \cap N\left(a_{3}\right) \cap B$ would induce a $C_{4}$, which is forbidden. This contradiction shows that $a_{1}$ and $a_{3}$ have no common neighbors in $B$ and therefore $N\left(a_{1}\right) \cap N\left(a_{3}\right)=A_{2} \cup\{x\}$.

From Lemma 1 it follows that $N(x)=\left(\bigcup_{i=0}^{4} N\left(a_{i-1}\right) \cap N\left(a_{i+1}\right)\right) \backslash\{x\}$. Therefore, $G$ can be described by $G \backslash\{x\}$ and five neighbors of $x$.

Assume now that a $\left(K_{1,3}, C_{4}\right)$-free graph $G$ contains a vertex $x$ whose neighborhood induces a graph obtained from a $W_{5}$ by duplicating some of its vertices. Again we denote $A=N(x)$ and $B=V(G) \backslash(A \cup\{x\})$. Also, let $C$ be the clique substituting the central vertex of the wheel and $A_{i}, i=0, \ldots, 4$ the cliques substituting the remaining vertices of the wheel. 
Lemma 2. Let $c \in C$, then $N(c) \cup\{c\}=A \cup\{x\}$.

Proof. It is clear that $N(c) \cup\{c\} \supseteq A \cup\{x\}$. In order to prove the lemma we need to show the reverse inclusion. Suppose to the contrary that there is a vertex $y$ in $B$ which is adjacent to $c$. The neighbors of $y$ in $A$ induce a complete graph, since otherwise $G$ would contain an induced $C_{4}$. Therefore, $N(y) \cap A \subseteq C \cup A_{i} \cup A_{i+1}$ for some index $i \in\{0, \ldots, 4\}$. Without loss of generality let $N(y) \cap A \subseteq C \cup A_{1} \cup A_{2}$, but then $c, y$ together with any two vertices $a_{0} \in A_{0}$ and $a_{3} \in A_{3}$ would induce a $K_{1,3}$. This contradiction proves the lemma.

According to Lemma 2 in the graph $G \backslash\{x\}$ the set $N(c) \cup\{c\}$ describes the neighborhood of vertex $x$ in the graph $G$. Therefore, to describe $G$ we need to know $G \backslash\{x\}$ and an arbitrary vertex $c \in C$.

Now let us denote by $\mathcal{Y}$ the class of $\left(K_{1,3}, C_{4}\right)$-free graphs in which the neighborhood of every vertex induces the complement of a chain graph. Obviously, this class is hereditary.

Lemma 3. The class Free $\left(K_{1,3}, C_{4}\right)$ is factorial if and only if $\mathcal{Y}$ is factorial.

Proof. If Free $\left(K_{1,3}, C_{4}\right)$ is factorial, then $\mathcal{Y}$ is factorial, because it is a proper subclass of $\operatorname{Free}\left(K_{1,3}, C_{4}\right)$ and it contains one of the minimal factorial classes (the class of complements of chain graphs).

Conversely, suppose that $\mathcal{Y}$ is a factorial class and let $G$ be an arbitrary $n$-vertex graph in Free $\left(K_{1,3}, C_{4}\right)$. If $G$ contains a vertex $x^{1}$ whose neighborhood induces either a $C_{5}$ or a wheel $W_{5}$, then we remove it from $G$ and record $x^{1}$ and (at most) five of its neighbors $a_{0}^{1}, a_{1}^{1}, a_{2}^{1}, a_{3}^{1}, a_{4}^{1}$ which allow us to describe the neighborhood of $x^{1}$. After this operation, we have a record containing at most 6 vertices and a graph $G^{1}$ obtained from $G$ by deleting $x^{1}$. If $G^{1}$ contains a vertex $x^{2}$ whose neighborhood induces either a $C_{5}$ or a wheel $W_{5}$, we repeat the procedure which leaves us with a record containing at most 12 vertices and a graph $G^{2}$ obtained from $G^{1}$ by deleting $x^{2}$. We repeat this procedure until we obtain a graph $G^{k}$ from $\mathcal{Y}$, where $k \leq n$ is the number of applications of the operation. The record

$$
x^{1}, a_{0}^{1}, a_{1}^{1}, a_{2}^{1}, a_{3}^{1}, a_{4}^{1}, \ldots, x^{k}, a_{0}^{k}, a_{1}^{k}, a_{2}^{k}, a_{3}^{k}, a_{4}^{k}, G^{k},
$$

completely describes the graph $G$ (i.e. allows to restore $G$ from the record). Therefore, the number of different records of type (2) equals the number of $n$-vertex labeled graphs in $\operatorname{Free}\left(K_{1,3}, C_{4}\right)$. Since $G^{k}$ is an $(n-k)$-vertex graph from a factorial class, an upper bound on this number can be estimated as follows:

$$
\sum_{k=0}^{n}\left(n\left(\begin{array}{l}
n \\
5
\end{array}\right) 5 !\right)^{k}(n-k)^{c(n-k)}<\sum_{k=0}^{n} 120^{k} n^{6 k+c(n-k)} \leq \sum_{k=0}^{n} 120^{k} n^{\max \{6, c\} n}<n^{c_{1} n}
$$

where $c_{1}$ is constant. From $(3)$ we conclude that $\operatorname{Free}\left(K_{1,3}, C_{4}\right)$ is a factorial class.

Lemma 3 allows us to focus in the rest of the section on graphs in the class $\mathcal{Y}$, which is precisely the class of $C_{4}$-free quasi-line graphs. 


\subsection{Quasi-line graphs without a square}

A graph is a quasi-line graph if the neighborhood of every vertex induces a co-bipartite graph. Obviously, the class of quasi-line graphs is superfactorial, since it contains all co-bipartite graphs. In this section, we study quasi-line graphs containing no $C_{4}$ as an induced subgraph and show that this class is factorial. A crucial role in our proof is played by the structural characterization of quasi-line graphs proposed by Chudnovsky and Seymour [9]. To describe this characterization we need to introduce a few definitions.

Circular interval graph.

Let $C$ be a circle, $V$ a finite set of points of $C$, and $I$ a set of intervals of $C$ (an interval is a proper subset of $C$ homeomorphic to $[0,1])$. Define $G$ to be the graph with vertex set $V$ and two vertices $u, v \in V(G)$ being adjacent if and only if $\{u, v\}$ is a subset of one of the intervals. We call the triple $(C, V, I)$ a circular interval representation of $G$. Any graph that admits a circular interval representation is called a circular interval graph. A special case of circular interval graphs are linear interval graphs which are defined in the same way with $C$ being a line instead of a circle.

Fuzzy circular interval graph.

A graph $G=(V, E)$ is a fuzzy circular interval if the following conditions hold:

1. There is a map $\phi$ from $V(G)$ to a circle $C$ (not necessarily injective).

2. There is a set of intervals $I$ of $C$, none including another, such that no point of $C$ is an endpoint of more than one interval so that:

(a) If two vertices $u$ and $v$ are adjacent, then $\phi(u)$ and $\phi(v)$ belong to a common interval.

(b) If two vertices $u$ and $v$ belong to a same interval, which is not an interval with endpoints $\phi(u)$ and $\phi(v)$, then they are adjacent.

In other words, for a fuzzy circular interval graph the pair $(\phi, I)$ completely describe adjacencies, except adjacencies for vertices $u$ and $v$ such that $I$ contains an interval with the endpoints $\phi(u)$ and $\phi(v)$. For such vertices adjacency is fuzzy. Note that if we require $\phi$ to be injective, the definition of a fuzzy circular interval graph would be equivalent to the definition of a circular interval graph. By replacing the circle $C$ with a line we obtain a definition of a fuzzy linear interval graphs

Strip

A strip is a triple $(G, a, b)$, where $G$ is a graph and $a, b$ are two designated simplicial vertices of $G$ called the ends of the strip. A strip $\left(G, v_{1}, v_{n}\right), n>1$ is called a linear interval strip if $G$ is a linear interval graph with vertices $v_{1}, \ldots, v_{n}$ listed in the order of their appearances in the linear interval representation of $G$. Fuzzy linear interval strips are defined analogously, provided that if $a, b$ are the endpoints of the strip then $\phi(a), \phi(b)$ are different from $\phi(v)$ for all other vertices $v$ of $G$. 


\section{Composition of strips}

The composition of two strips $(G, a, b)$ and $\left(G^{\prime}, a^{\prime}, b^{\prime}\right)$ is the graph obtained from the union of $G \backslash\{a, b\}$ and $G^{\prime} \backslash\left\{a^{\prime}, b^{\prime}\right\}$ by adding to it all possible edges between $N(a)$ and $N\left(a^{\prime}\right)$ and between $N(b)$ and $N\left(b^{\prime}\right)$.

Let $G_{0}$ be a disjoint union of complete graphs with an even number of vertices whose vertex set is partitioned into pairs of nonadjacent vertices, i.e. $V\left(G_{0}\right)=\left\{a_{1}, b_{1}, \ldots, a_{k}, b_{k}\right\}$ with $a_{i}$ being nonadjacent to $b_{i}$ for each $i=1, \ldots, k$. Also, for $i=1, \ldots, k$, let $\left(G_{i}^{\prime}, a_{i}^{\prime}, b_{i}^{\prime}\right)$ be a collection of $k$ strips that are vertex-disjoint, also from $G_{0}$. For $i=1, \ldots, k$, let $G_{i}$ be the graph obtained by composing $\left(G_{i-1}, a_{i}, b_{i}\right)$ with $\left(G_{i}^{\prime}, a_{i}^{\prime}, b_{i}^{\prime}\right)$. The resulting graph $G_{n}$ is called a composition of the strips $\left(G_{i}^{\prime}, a_{i}^{\prime}, b_{i}^{\prime}\right)(1 \leq i \leq k)$.

Chudnovsky and Seymour proved the following structural result in [9].

Theorem 8. A connected quasi-line graph $G$ is either a fuzzy circular interval graph or a composition of fuzzy linear interval strips.

\subsubsection{The structure of quasi-line graphs without $C_{4}$}

In this section we show that the class of quasi-line graphs without a $C_{4}$ is obtained from the class of quasi-line graphs by excluding the fuzziness from the definition.

Let $G$ be fuzzy circular interval graph with a representation $(\phi, I)$. If $[p, q]$ is an interval of $I$ such that $\phi^{-1}(p)$ and $\phi^{-1}(q)$ are both non-empty, then the pair of cliques $\left(\phi^{-1}(p), \phi^{-1}(q)\right)$ is called a fuzzy pair, where $\phi^{-1}(p)$ denotes the clique $\{v \in V(G) \mid \phi(v)=$ $p\}$. The following Lemma was proved in [10].

Lemma 4. Let $G$ be a fuzzy circular interval graph with a representation $(\phi, I)$. If no fuzzy pair contains an induced $C_{4}$, then $G$ is a circular interval graph.

As an immediate corollary from this lemma, we make the following conclusion.

Corollary 1. If $G$ is a fuzzy circular interval graph containing no $C_{4}$ as an induced subgraph, then $G$ is a circular interval graph.

Also, since a fuzzy linear interval graph is a special case of a fuzzy circular interval graph, we conclude the following.

Corollary 2. If $(G, a, b)$ is a fuzzy linear interval strip containing no $C_{4}$ as an induced subgraph, then $(G, a, b)$ is a linear interval strip.

Now we show that if $H$ is a composition of strips and $H$ is a $C_{4}$-free graph, then each strip in the composition also is $C_{4}$-free. Obviously, it suffices to prove this statement only for two strips.

Lemma 5. Let $H$ be the composition of two fuzzy linear interval strips $\left(G_{1}, a_{1}, b_{1}\right)$ and $\left(G_{2}, a_{2}, b_{2}\right)$. If $\mathrm{H}$ is $C_{4}$-free, then both strips are $C_{4}$-free. 
Proof. Suppose without loss of generality that $G_{1}$ contains an induced $C_{4}$. Since $a_{1}$ and $b_{1}$ are simplicial, they do not belong to any induced $C_{4}$. Therefore, $G_{1} \backslash\left\{a_{1}, b_{1}\right\}$ also contains an induced $C_{4}$. By definition, $G_{1} \backslash\left\{a_{1}, b_{1}\right\}$ is an induced subgraph of $H$. Therefore, $H$ also contains an induced $C_{4}$, which contradicts the assumption.

Combining this lemma with Corollaries 1 and 2 and Theorem 8 we obtain the following structural characterization of $C_{4}$-free quasi-line graphs.

Theorem 9. A connected quasi-line graph $G$ without an induced $C_{4}$ is either a circular interval graph or a composition of linear interval strips.

\subsubsection{The number of $n$-vertex quasi-line graphs without $C_{4}$}

In this section we show that the class of $C_{4}$-free quasi-line graphs is factorial. The lower bound follows from the fact that this class contains $P^{1}$ (one of the minimal factorial classes). Therefore, we only need to prove an upper bound. To this end, we may restrict ourselves to connected graphs only.

We start by estimating the number of circular interval graphs. It is known (see e.g. [9]) that this class is a proper subclass of the class of circular arc graphs. A graph $G$ is a circular arc graph if it is the intersection graph of a set $I=\left\{I_{1}, \ldots, I_{n}\right\}$ of intervals (arcs) on a circle $C$, i.e. if there exists a one-to-one correspondence between the vertices of $G$ and the intervals of $I$ such that two vertices of $G$ are adjacent if and only if the respective intervals intersect (without loss of generality, we may assume that no two intervals of $I$ share the same endpoint). The pair $(C, I)$ is called a circular arc model of $G$. We call two circular arc models different if they define different labeled graphs and equivalent otherwise.

Lemma 6. The class of circular arc graphs is factorial.

Proof. The lower bound follows from the fact that this class contains $P^{1}$. For the upper bound, let $G$ be an $n$-vertex circular arc graph and $(C, I)$ a circular arc model representing $G$ with $I=\left\{\left[a_{i}, b_{i}\right], i=1, \ldots, n\right\}$. Starting from $a_{1}$, we write out the labels of the endpoints of intervals in the order they appear on the circle clockwise, which results in a sequence

$$
\pi_{I}=\left[c_{1}, c_{2}, \ldots, c_{2 n}\right],
$$

where every element of the sequence is either $a_{i}$ or $b_{i}$ for some value of $i$. In particular, $c_{1}=a_{1}$. It is clear that if $\pi_{I}=\pi_{I^{\prime}}$, then the models $(C, I)$ and $\left(C, I^{\prime}\right)$ are equivalent, i.e. they represent the same labeled graph. Therefore, the number of different circular arc models does not exceed the number of different sequences of type (4), which is $(2 n)$ !. Therefore, the class of circular arc graphs is factorial.

Corollary 3. The class of circular interval graphs and the class of linear interval graphs are factorial.

Proof. Corollary follows from the fact that both classes are subclasses of the class of circular arc graphs and both of them contain $P^{1}$ (one of the minimal factorial classes). 
Now we turn to estimating the number of different labeled $n$-vertex compositions of linear interval strips. Without loss of generality, we assume that every strip contains at least 3 vertices, since otherwise it adds nothing to the composition and hence can be ignored.

Any $n$-vertex composition of $k$ strips is completely determined by

- a $2 k$-vertex labeled graph $G_{0}$, which is a disjoint union of cliques, given together with a partition of its vertices into pairs $\left\{a_{1}, b_{1}, \ldots, a_{k}, b_{k}\right\}$,

- an ordered list of $k$ labeled strips $\left(G_{i}^{\prime}, a_{i}^{\prime}, b_{i}^{\prime}\right)(i=1, \ldots, k)$.

Note that $k \leq n$, since each strip adds at least one vertex to the resulting graph. We denote the number of vertices in the $i$-th strip by $n_{i}=t_{i}+2$, where $t_{i}$ is the number of vertices the $i$-th strip contributes to the resulting graph. Therefore, $\sum_{i=1}^{k} t_{i}=n$ and hence $\sum_{i=1}^{k} n_{i}=n+2 k \leq 3 n$.

Let $G=(V, E)$ be a $n$-vertex composition of $k$ strips. The strips define an ordered partition of $V$ into $k$ nonempty subsets. We fix this partition and estimate the number of ways to compose $G$ based on this partition. There are at most $(2 k)^{2 k}$ ways to create $G_{0}$ and at most $(2 k)^{2 k}$ ways to pair its vertices. If $G$ is $C_{4}$-free, then by Theorem 9 every strip in the composition is linear interval. By Corollary 3, the class of linear interval graphs is factorial and hence the number of labeled linear interval strips on $n_{i}$ vertices does not exceed $n_{i}^{c n_{i}}$ for a constant $c$. Therefore, for a fixed ordered partition of $V$ into $k$ nonempty subsets, there are at most

$$
(2 k)^{4 k} \prod_{i=1}^{k} n_{i}^{c n_{i}} \leq(2 n)^{4 n} n^{\sum_{i=1}^{k} c n_{i}} \leq n^{11 c n}
$$

ways to compose $G=(V, E)$.

The number of different ordered partitions of $V$ into $k$ subsets does not exceed $k^{n}$ and therefore the total number of labeled $n$-vertex compositions of linear interval strips is bounded by

$$
\sum_{k=1}^{n} n^{11 c n} k^{n} \leq n^{(11 c+1) n+1}<n^{c_{1} n},
$$

for a constant $c_{1}$. This fact together with Corollary 3 and Theorem 9 imply the following conclusion.

Lemma 7. The class of $C_{4}$-free quasi-line graphs is factorial.

Now from Lemmas 3 and 7 follows the main result of the section.

Theorem 10. The class Free $\left(K_{1,3}, C_{4}\right)$ is factorial. 


\section{References}

[1] V.E. Alekseev, Range of values of entropy of hereditary classes of graphs, Diskret. Mat. 4 (1992), no. 2, 148-157 (in Russian; translation in Discrete Mathematics and Applications, 3 (1993), no. 2, 191-199).

[2] V. Alekseev, On lower layers of the lattice of hereditary classes of graphs, Diskretn. Anal. Issled. Oper. Ser. 1 Vol. 4 ,no. 1, (1997), 3-12 (in Russian).

[3] P. Allen, Forbidden induced bipartite graphs, Journal of Graph Theory, 60 (2009) 219-241.

[4] P. Allen, V. Lozin and M. Rao, Clique-width and the speed of hereditary properties, Electronic Journal of Combinatorics, 16 (2009) Research Paper 35.

[5] J. Balogh, B. Bollobás and D. Weinreich, The speed of hereditary properties of graphs, J. Combin. Theory B, 79 (2000) 131-156.

[6] J. Balogh, B. Bollobás and D. Weinreich, A jump to the Bell number for hereditary graph properties, J. Combin. Theory Ser. B 95 (2005) 29-48.

[7] A. BrandstäDt, J. Engelfriet, H.-O. Le and V.V. Lozin, Clique-Width for 4-Vertex Forbidden Subgraphs Theory of Computing Systems, 34 (2006) 561-590.

[8] P. Cameron, T. Prellberg and D. Stark, Asymptotic enumeration of 2-covers and line graphs, Discrete Mathematics, 310 (2010) 230-240.

[9] M. Chudnovsky and P. Seymour, The structure of claw-free graphs, London. Math. Soc. Lecture Note Series, 327 (2005) 153-171.

[10] F. Eisenbrand, G. Oriolo, G. Stauffer and P. Ventura, The stable set polytope of quasi-line graphs, Combinatorica, 28 (2008) 45-67.

[11] T. KLOKS, $K_{1,3}$-free and $W_{4}$-free graphs, Information Processing Letters, 60 (1996) $221-223$.

[12] S. Norine, P. Seymour, R. Thomas and P. Wollan, Proper minor-closed families are small, J. Combinatorial Theory B 96 (2006) 754-757.

[13] E.R. Scheinerman and J. Zito, On the size of hereditary classes of graphs, J. Combin. Theory B 61 (1994) 16-39.

[14] J.P. SpInRAD, Nonredundant 1's in $\Gamma$-free matrices, SIAM J. Discrete Math. 8 (1995) 251-257.

[15] S. WAGON, A bound on the chromatic number of graphs without certain induced subgraphs, J. Combinatorial Theory B 29 (1980) 345-346. 